\title{
Review Article \\ Contralateral Risk-Reducing Mastectomy: Review of Risk Factors and Risk-Reducing Strategies
}

\author{
N. N. Basu, ${ }^{1,2}$ L. Barr, ${ }^{1}$ G. L. Ross, ${ }^{3}$ and D. G. Evans ${ }^{1,4}$ \\ ${ }^{1}$ Nightingale and Genesis Prevention Centre, University Hospital South Manchester, Southmoor Road, Manchester M23 9LT, UK \\ ${ }^{2}$ Department of Breast Surgery, Queen Elizabeth Hospital, Birmingham B15 2TH, UK \\ ${ }^{3}$ The Institute of Cancer Sciences, The University of Manchester, Oxford Road, Manchester M13 9PL, UK \\ ${ }^{4}$ University of Manchester Department of Genomic Medicine, Institute of Human Development, St. Mary's Hospital, Oxford Road, \\ Manchester M13 9WL, UK \\ Correspondence should be addressed to N. N. Basu; naren_basu@hotmail.com
}

Received 20 August 2014; Revised 2 January 2015; Accepted 9 January 2015

Academic Editor: Kazuhiro Yoshida

Copyright (C) 2015 N. N. Basu et al. This is an open access article distributed under the Creative Commons Attribution License, which permits unrestricted use, distribution, and reproduction in any medium, provided the original work is properly cited.

Rates of contralateral risk-reducing mastectomy have increased substantially over the last decade. Surgical oncologists are often in the frontline, dealing with requests for this procedure. This paper reviews the current evidence base regarding contralateral breast cancer, assesses the various risk-reducing strategies, and evaluates the cost-effectiveness of contralateral risk-reducing mastectomy.

\section{Introduction}

Breast cancer is the most common cancer in women worldwide, with 1.7 million new cases diagnosed in $2012[1,2]$. It accounts for $25 \%$ of all cancers in women and in the UK; it is estimated that 1 in $8-10$ women will develop breast cancer [3] during their lifetime.

Breast cancer survivorship has improved as a result of early detection and advancing treatment modalities [3]. As such, management of this group of women requires healthcare professionals to be familiar with additional risks factors so that timely recommendations may be made on surveillance or risk-reducting strategies.

Once diagnosed with breast cancer, these women will have an increased risk of developing a contralateral, metachronous breast cancer [4]. The level of this risk is multifactorial, dependent on tumour biology, adjuvant treatment, and oncogenetics.

There is ongoing interest in contralateral breast cancer (CBC). Surveillance, Epidemiology, and End Results Program (SEER) data from the US [5] has confirmed a $150 \%$ increase in rates of contralateral risk-reducing mastectomy (CRRM) over the last decade, although this may not be the case in Europe [6]. This rise is somewhat surprising, given that rates of $\mathrm{CBC}$ are decreasing (due to endocrine therapy especially with aromatase inhibitors) and may be a reflection of a heightened perceived risk in this vulnerable group of women. Clinicians are at the frontline of many of these complex decisions and need to make evidence-based recommendations.

We examine the multiple risk factors known to contribute to developing CBC. Survival data are analysed and the contribution of the following risk factors is discussed: gene mutation and family history, histology, ER status, and HER2 status. We evaluate the different risk-reducing strategies (surgery and chemoprevention) and their efficacy and cost and finally consider the patient's perspective. We have included chemoprevention using antiendocrine treatment in the section of ER status as this is relevant to this section.

This review aims to serve as an aide-memoire for clinicians to refer to when counselling women on CBC.

\section{Incidence of $\mathbf{C B C}$}

High-risk patients include breast cancer sufferers with known genetic mutations (BRCA1/2, TP53) and a significant family history. BRCA1/2 mutation carriers have a $\mathrm{CBC}$ risk of 2$3 \%$ per annum [8]. This is likely to be higher in TP53 patients, though there is limited data on this group of patients [9]. This heightened risk, particularly in women diagnosed 
with their primary cancer before 40 years, lasts for at least 20 years. Risk-reducing bilateral salpingo-oophorectomy (RRBSO) and menopause before the age of $40[8,10,11]$ are protective factors.

The remainder of patients without a known genetic mutation or significant family history represent the majority of breast cancer sufferers. SEER historical data (1973-1996) quote actuarial incidence rates of $\mathrm{CBC}$ in this group of women at $5,10,15$, and 20 years were $3 \%, 6.1 \%, 9.1 \%$, and $12 \%$, respectively, amounting to $0.6 \%$ per annum [4]. This level of risk is likely to be outdated with several studies supporting a global decrease in $\mathrm{CBC}$, with almost a $30 \%$ reduction in parts of Europe over the last 10-year period [4]. This trend is likely to be a reflection of successful adjuvant treatment, in particular antiendocrine treatment.

\subsection{Risk Factors}

2.1.1. Gene Mutation. The two most commonly studied breast cancer susceptibility genes are BRCA1 and BRCA2. Mutations in these tumour suppressor genes confer an up to $80-90 \%$ lifetime risk of developing breast cancer [12-14].

These women have a significant $\mathrm{CBC}$ risk once diagnosed with breast cancer.

The risk, up to age 70 years, of a CBC in BRCA1 mutation carriers has been estimated to be above 60\% [15] and in BRCA2 mutation carriers slightly lower at around 50\% [12]. However, a recent prospective study [16] has shown that these risks may be even higher at $83 \%$ and $63 \%$, respectively, representing higher risks in the modern era particularly for $B R C A 1$ where the majority of tumours would not receive endocrine therapy.

Several studies have evaluated further CBC risk factors within $B R C A 1 / 2$ mutation carriers $[8,10,11]$. Early age of first breast cancer diagnosis ( $<50$ years) with increasing numbers of first-degree relatives with breast cancer at a young age heightens that risk. Protective factors that reduce $\mathrm{CBC}$ risk include the use of tamoxifen (HR 0.59; 95\% CI 0.31-1.01) $[8,17]$ and oophorectomy (HR $0.44 ; 95 \%$ CI $0.21-0.91$ ), with additional benefit of oophorectomy prior to the age of 49 years (HR 0.24; 95\% CI 0.07-0.77).

Breast cancer remains the most common malignancy in women harbouring a TP53 mutation in Li-Fraumeni syndrome. These mutation carriers will have a nearly $100 \%$ lifetime risk of developing cancer with the vast majority developing breast cancer by the age of 46 [18].

Given the rarity of this condition, there is very limited data on $\mathrm{CBC}$ risk. Evans et al. [9] studied women under the age of 30 years with breast cancer and found that rates of $\mathrm{CBC}$ were approximately 2-3\% annually in all mutations carriers (TP53 and BRCA1/2), although only 11 TP53 mutation carriers were included in their extended analysis. It is possible that adjuvant endocrine and anti-HER2 treatment will influence CBC risk in TP53 mutation carriers as the majority of these patients are ER and HER2 positive [19]. Efforts are underway to assess this risk further.

2.1.2. Family History. A positive family history of breast cancer increases the risk of $\mathrm{CBC}$ although this risk pattern is complex. Vichapat et al. [7] studied 8478 women with breast cancer over a 31-year period (1975-2006) and found that there was a 2.8 -fold increase in relative risk with a positive family history of breast cancer. Subgroup analysis revealed that the highest risks were those with a first- and seconddegree relative (RR 2.33) followed by first-degree alone (RR 1.38 ) and second- or third-degree (RR 1.13). Numerous firstdegree relatives will confer an even higher risk.

The WECARE study (Women's Environment Cancer and Radiation Epidemiology Study) conducted a populationbased case control study comparing asynchronous bilateral breast cancer patients (case) against unilateral breast cancer (control) patients [20]. They confirmed that the risk of CBC in noncarriers of $B R C A 1 / 2$ mutation with a family history was highest in women diagnosed at an earlier age with their index breast cancer ( $<45$ years), those with a young firstdegree relative, particularly with bilateral disease. The 10-year cumulative CBC risk stratified by age was $6.7 \%$ ( $50-54$ years), $9.0 \%$ (40-44 years), and $14.7 \%$ (30-34 years).

A study from the Mayo Clinic [21] followed up 745 women with breast cancer and a positive family history who underwent a CRRM between 1960 and 1993. They had predicted (without CRRM) 106 CBCs in the premenopausal group and $50 \mathrm{CBCs}$ in the postmenopausal group. CRRM had resulted in an approximate 95\% reduction in relative risk as only 6 and 2 actual CBCs occurred in pre- and postmenopausal women, respectively.

2.1.3. Histology of Index Breast Cancer. The Vichapat et al. study [7] assessed histological type and found no significant increase in CBC in those with lobular breast cancer. This is an interesting finding given that lobular breast cancer has been shown previously to be an independent predictor of increased CRRM rates [22] and possibly arises from previous studies that have shown an association of lobular cancer and $\mathrm{CBC}$ [23].

High grade of primary tumours (RR 1.3 for Grade 3 cancer compared to Grade 1), increasing size ( $<2 \mathrm{~cm}$ RR $1.0,2-5 \mathrm{~cm}$ RR 1.51, and $>5 \mathrm{~cm}$ RR 1.89), and number of positive lymph nodes (non-RR 1.0, 4-9 RR 1.12, and >10 RR 1.62) were all shown to be important risk factors [7]. Table 1 summarises the known risk factors for developing contralateral breast cancer while Table 2 shows the risk reduction strategies of CBC.

2.1.4. HER2 Status and Anti-HER2 Treatment. Up to $30 \%$ of breast cancers [24] express HER2 receptor tyrosineprotein kinase. Use of the monoclonal antibody trastuzumab (Herceptin) has been shown to improve disease-free survival [25]. The HERA study (Herceptin Adjuvant Trial) recently reported outcomes after a 4-year follow-up [26]. In the observation group there were $19 / 320$ (1.1\%) CBCs compared to $14 / 251(0.8 \%)$ in the trastuzumab group. Although this represents a small reduction in CBC in women treated with trastuzumab, its clinical application for risk reduction of $\mathrm{CBC}$ remains debatable.

Saltzman et al. [27] performed a case-control study of 29,126 women using the Cancer Surveillance System (CSS) cancer registry. They were able to show that women with 
TABLE 1: Risk factors for developing CBC (estimated annual risk) $[4,7]$.

\begin{tabular}{cc}
\hline Estimated & Relative \\
annual & risk-multivariate \\
risk (\%) [4] & $(95 \% \mathrm{CI})[7]$ \\
\hline
\end{tabular}

\section{Patient factors}

Age at first diagnosis

$\begin{array}{ll}<30 \text { yrs } & 0.5-1.3 \\ 40-50 \text { yrs } & \\ \text { ER +ve } & 0.2-0.3 \\ \text { ER -ve } & 0.4-0.5\end{array}$

Gene mutation

$\begin{array}{ll}B R C A 1 & 2.0-3.0 \\ B R C A 2 & 2.0-3.0\end{array}$

Family history

$$
\text { None }
$$

First- and second-degree

First-degree

Second- or third-degree

Tumour factors

Size

$$
<2 \mathrm{~cm}(\mathrm{~T} 1)
$$

2-5 cm (T2)

$>5 \mathrm{~cm}(\mathrm{~T} 3)$

LN status

None

$1-3$

$4-9$

$>10$

Histology

Ductal

Lobular

ER status

ER positive

ER negative

HER2 status

HER2 positive

HER2 negative

HER2 overexpression (ER negative/HER2 positive) and those with triple negative cancer (ER negative, PR negative, and HER2 negative) had a 2.0-fold and 1.4-fold increased risk of developing $\mathrm{CBC}$, respectively. Therefore, in addition to having a higher risk of recurrent disease and death, this subgroup of patients will have an elevated risk of CBC and surveillance strategies need to be considered in monitoring this cohort.

2.1.5. ER Status and Chemoprevention. Several studies (ATAC, IBIS I, IBIS II, and STAR) have confirmed that antihormonal agents (SERM and aromatase inhibitors) given to high-risk women for up to 10 years can reduce
TABLE 2: Risk reduction of CBC associated with chemoprevention and surgery.

\begin{tabular}{ll}
\hline & $\begin{array}{c}\text { Risk reduction } \\
(95 \% \mathrm{CI})\end{array}$ \\
\hline $\begin{array}{l}\text { Chemoprevention } \\
\text { Antiendocrine }\end{array}$ & \\
Tamoxifen in BRCA1/2 mutation carriers & OR $0.5(0.3-0.9)$ \\
Tamoxifen in noncarriers & $50 \%$ risk reduction \\
Aromatase inhibitors in noncarriers & $70 \%$ risk reduction \\
Chemotherapy & \\
Chemo versus no chemo & RR 0.6 $(0.4-0.8)$ \\
Surgery & $20-$ year survival \\
CRRM & benefit \\
CRRM in BRCA1/2 mutation carriers & $14.9 \%$ \\
CRRM in nonmutation carriers & $<1 \%$ \\
\hline
\end{tabular}

the incidence of $\mathrm{CBC}$ and primary breast cancer [28]. Women with hormone sensitive index breast cancers are routinely offered antihormonal agents as part of their adjuvant treatment and can expect an up to $50 \%$ reduction in their risk of developing a $\mathrm{CBC}$ with recent studies showing favouring aromatase inhibitors over tamoxifen (ATAC) in the postmenopausal setting [29]. Women considering antihormonal agents need to be appraised of significant adverse effects including thromboembolic phenomenon, osteoporosis, and uterine carcinoma.

Recently, Gronwald et al. [17] were able to confirm previous studies showing an approximately $50 \%$ reduction in CBC risk in BRCA1/2 mutation carriers who took Tamoxifen following their index breast cancer. Of interest was the similar risk-reduction of a short period of Tamoxifen $(<1$ year $)$ compared to longer use ( $>4$ years). This has implications on women who have concerns over the side effect profile of long term Tamoxifen use and may rationalise the short-term use of this drug.

2.1.6. Chemotherapy. Cytotoxic chemotherapy agent recommended as adjuvant or neoadjuvant treatment of primary breast cancer has been shown to reduce the risk of $\mathrm{CBC}$. The Early Breast Cancer Trialists Group (EBCTG) showed a marginal reduction in the incidence of $\mathrm{CBC}$ over a 15 -year follow-up period [30], which was more definite in women under the age of 50 years.

The WECARE group carried out a case-control study [31] and found that chemotherapy use was associated with a 35$40 \%$ reduction in risk of CBC in women under the age of 55 and that this protective effect lasted up to 10 years. In addition, those who became postmenopausal within 1 year of diagnosis had the greatest risk reduction.

PARP inhibitors and their effect on triple negative and $B R C A 1 / 2$ mutation related breast cancers are the subject of much interest [32]. Initial reports from proof-of-concept trials [33] have confirmed their safety and efficacy, and phase III studies with an extended follow-up may determine 
whether these targeted therapy modalities affect contralateral breast cancer risk.

\subsection{Contralateral Risk-Reducing Mastectomy}

2.2.1. Survival. The aftermath of Angelina Jolie's announcement of her bilateral risk-reducing mastectomy (BRRM) has raised public awareness of risk-reducing surgery [34, 35]. Several studies have confirmed a survival benefit in high-risk patients $(B R C A 1 / 2$ and $\mathrm{FH})$ undergoing BRRM and CRRM [36-39]. Our own experience [39] compared 105 female $B R C A 1 / 2$ mutation carriers with unilateral breast cancer to matched mutation carriers who did not undergo CRRM. The overall 10 -year survival was $89 \%$ in the CRRM group compared to $71 \%$ in the non-CRRM group $(P<0.001)$, which was independent of RRBSO. This is in contrast to van Sprundel et al. [40] who found that, after adjusting for RRBSO, there was no overall survival benefit from CRRM. Metcalfe et al. [38] followed a similar group of 390 BRCA1/2 mutation carriers and found that at 20 years the survival rate of those who underwent CRRM was 88\% (CI 83-93\%) compared to $65 \%$ (CI 59-73\%) who did not undergo CRRM.

The survival benefit in non-BRCA mutation patients is less clear. Younger women under the age of $49[41,42]$ with ER -ve disease are likely to have an improved disease-specific survival, which is thought to be due to a higher baseline risk of CBC. A clear survival benefit on the remainder of patients seems less likely [37]. Portschy et al. [43] used a Markov model to compare survival outcomes between CRRM and non-CRRM in non-BRCA patients. They estimated a less than $1 \%$ absolute 20 -year survival benefit from CRRM amongst all age groups, ER status, and cancer stage groups.

2.2.2. Breast Reconstruction. Several studies have shown that access to immediate breast reconstruction positively affects the decision for CRRM [44, 45]. Recently, Ashfaq et al. [45] identified 102,674 patients (2004-2008) with a diagnosis of DCIS (15\%) or invasive breast cancer (85\%) from SEER registry data. Those undergoing mastectomy were 3 times more likely to request CRRM if offered immediate reconstruction. Overall, $16 \%$ of all patients underwent CRRM with a significant proportion undergoing reconstruction $(46 \%$, $P<0.001)$. Similar proportions of patients underwent implant-based reconstruction (36\%) and tissue-based reconstruction (37\%). There was a trend of increasing numbers of reconstructions during this time period, and Caucasian women, under the age of 45 years with a diagnosis of a nodenegative lobular carcinoma or DCIS, were more likely to choose reconstruction.

Women undergoing reconstruction following CRRM are 1.5 times more likely to have a major complication requiring hospitalisation or reoperation [46] compared to unilateral mastectomy. Limited data is available comparing CRRM and reconstruction with unilateral mastectomy and reconstruction. Crosby et al. [47] assessed 497 patients undergoing CRRM with reconstruction and concluded that a third of patients experiencing at least one complication may not have developed a complication if they had only had a mastectomy and reconstruction of their index side.
2.2.3. Sentinel Lymph Node Biopsy (SLNB). SLNB at the time of risk-reducing surgery remains controversial. A recent meta-analysis of 1251 patients [48] showed that $1.7 \%(n=$ 21) of women undergoing RRM harboured occult invasive cancer in the mastectomy specimen. Of these 21 patients, the SLN was positive in only $4 / 21$ patients and negative in the remainder (17/21). This was higher in women with advanced cancer in the contralateral breast. Overall, $2.8 \%$ $(n=36)$ of women benefited from SLNB that included 19 cases of a positive SLNB result requiring completion axillary surgery and 17 women who had invasive disease in the mastectomy specimen but a negative SLNB, thus avoiding further axillary surgery. This is offset against the 5\% lymphoedema rate associated with SLNB [49]. Kuwajerwala et al. [50] retrospectively assessed 170 patients undergoing CRRM and found that of the $21.8 \%$ who had a SLNB at the surgeon's discretion, none had positive SLNB.

2.2.4. Cost. Health care economics contribute to the decision making process. Cost-effectiveness with life expectancy gains is well established in the setting of bilateral RRM in women harbouring BRCA1/2 mutations [51, 52]. Few studies have looked at this in the setting of CRRM [53-55]. Deshmukh et al. [54] analysed matched groups (CRRM and nonCRRM) and showed that CRRM significantly increases shortterm healthcare cost by $\$ 7,749$. In addition, women who had a reconstruction and in particular a delayed type had significantly higher cost associated as well as those who had HER2 positive disease and received radiotherapy.

In contrast, Roberts et al. [55] found that CRRM was costeffective in the prevention of $\mathrm{CBC}$ in women under the age of 50 years. From their decision tree-model, they concluded that 68,000 women under the age of 50 years would have been diagnosed with early breast cancer in 2010. If all women had undergone CRRM, savings of $\$ 19$ million would have been made to avoid 3,900 contralateral breast cancers that would have developed over the next 10 years. Their CRRM group had 0.2 quality-adjusted life years (QUALYs) less than the nonCRRM that may have been accounted for by complications of reconstruction. They highlighted a potential greater benefit of CRRM in ER - ve disease compared to ER + ve disease, given that the latter would receive adjuvant endocrine treatment, shown to reduce $\mathrm{CBC}$.

Zendejas et al. [53] used a Markov model to compare cost-effectiveness in women undergoing CRRM compared to routine surveillance (including annual mammography). They found that CRRM prior to the age of 70 years was costeffective and in particular in those who were BRCA-positive.

Currently in the UK, there are no funding restrictions within the National Health Service on CRRM. Breast cancer patients can choose between delayed and immediate (performed at the same time as the therapeutic mastectomy) without financial scrutiny provided that there is backing from the relevant clinicians.

2.2.5. Patient's Perspective. One of the main driving forces for CRRM is patients' worry and anxiety about developing another breast cancer and having to undergo further treatment including chemotherapy. This is often the most 
difficult component to assess, as the psychology behind it is multifactorial. A recent US study reported that $68.9 \%$ of patients undergoing CRRM did not have genetic or familial risk factors for $\mathrm{CBC}$ [56] and that the main driving force for this was worry about recurrence. Patients overestimate their risk of contralateral breast cancer [57] and in doing so can compound their anxiety.

A recent study [58] assessed the perspective from 60 consecutive patients choosing CRRM. In almost all cases, requests for CRRM were instigated by the patient and every patient unambiguously wanted CRRM. Patients responded to risk in an "all or nothing" manner and the majority did not objectively quantify this risk. The risk assessment in those that did quantify risk had little role in their decision for surgery. The authors concluded that "patients' subjective sense of vulnerability overwhelmed their appreciation of risk so that, so that regardless of level of risk of $\mathrm{CBC}$, they found this risk intolerable and felt that only CRRM could reduce it." This showed that a rate limiting factor will be the availability of immediate reconstruction and if this is not made possible for patients as part of their primary surgical treatment rates of CRRM are likely to be lower.

\section{Discussion}

Rates of CRRM have increased in the US, a trend that in the future other countries may follow. This is of concern given that the actual incidence on $\mathrm{CBC}$ is on the decrease as a result of successful adjuvant treatments.

CBC risk assessment is multifactorial and may be assessed in a multidisciplinary setting. The most significant risk factors are gene mutation status and significant family history, which can result in at least a fourfold increase in $\mathrm{CBC}$ risk. Patients harbouring a $B R C A 1 / 2$ mutation have an approximate 2$3 \%$ annual incidence on developing CBC. In nonmutation carriers with a family history, young age at first diagnosis and first-degree relative are particularly strong risk factors.

Tumour biology is important. The ER status is of particular importance given that approximately $70 \%$ of all breast cancers are hormone sensitive. Risk reduction with antiendocrine treatment is approximately $50 \%$ with tamoxifen and $70 \%$ with aromatase inhibitors. As predicted, women with ER negative breast cancer have an increased risk of CBC. The use of cytotoxic agents and targeted treatments (e.g., Herceptin) marginally reduces $\mathrm{CBC}$, with the greatest benefit for young women having chemotherapy.

CRRM offers the greatest risk reduction of $\mathrm{CBC}$, up to $95 \%$ in women with a family history. Survival benefits are conferred on those high-risk patients with a $B R C A 1 / 2$ mutation. There is no survival benefit in the non-high-risk group.

Access to immediate breast reconstruction positively affects a woman's decision to opt for CRRM, with a significant number experiencing operative complications. Occult disease in the CRRM specimen occurs in less than $2 \%$ of women with no clear evidence to support sentinel lymph node biopsy.

Survival benefits and cost-effectiveness are seen in those at the highest risk of $\mathrm{CBC}$ (gene mutation carriers) and these patients are likely to benefit most from CRRM.
The assessment of $\mathrm{CBC}$ risk is multifactorial and may be assessed in a multidisciplinary setting. Arrington et al. [22] showed that surgeon and patient characteristics determine CRRM and include independent factors like young patient age ( $<40$ years), large tumour size $(>5 \mathrm{~cm})$, lobular histology, positive family history, multicentric disease, and female surgeon. In addition, patients' anxiety about developing another breast cancer and going through subsequent treatment is a real entity, albeit difficult to quantify.

All clinicians treating breast cancer patients should be familiar with $\mathrm{CBC}$ risk and have the opportunity to discuss the various options including CRRM, chemoprevention, and routine surveillance. The multidisciplinary team is invaluable in guiding women based on objective assessment of genetic and family history, tumour biology, and psychological support.

It is becoming apparent that women seeking CRRM are categorised into different risk groups [59] and that clinicians are faced with differing challenges when managing these different groups. This review has focused on the different risk factors and risk-reducing strategies to give clinicians a comprehensive overview of the current literature.

\section{Conflict of Interests}

The authors declare that there is no conflict of interests regarding the publication of this paper.

\section{References}

[1] D. M. Parkin, P. Pisani, and J. Ferlay, "Estimates of the worldwide mortality from 25 cancers in 1990," International Journal of Cancer, vol. 83, no. 1, pp. 18-29, 1999.

[2] World Cancer Research Fund International, Breast Cancer Statistics, 2012, http://www.wcrf.org/int/cancer-facts-figures/ data-specific-cancers/breast-cancer-statistics.

[3] Cancer Research UK, 2014, http://cancerresearchuk.org/cancer.

[4] I. M. Lizarraga, S. L. Sugg, R. J. Weigel, and C. E. H. ScottConner, "Review of risk factors for the development of contralateral breast cancer," The American Journal of Surgery, vol. 206, no. 5, pp. 704-708, 2013.

[5] T. M. Tuttle, E. B. Habermann, E. H. Grund, T. J. Morris, and B. A. Virnig, "Increasing use of contralateral prophylactic mastectomy for breast cancer patients: a trend toward more aggressive surgical treatment," Journal of Clinical Oncology, vol. 25, no. 33, pp. 5203-5209, 2007.

[6] U. Güth, M. E. Myrick, C. T. Viehl, W. P. Weber, A. M. Lardi, and S. M. Schmid, "Increasing rates of contralateral prophylactic mastectomy-a trend made in USA?" European Journal of Surgical Oncology, vol. 38, no. 4, pp. 296-301, 2012.

[7] V. Vichapat, C. Gillett, I. S. Fentiman, A. Tutt, L. Holmberg, and M. Lüchtenborg, "Risk factors for metachronous contralateral breast cancer suggest two aetiological pathways," European Journal of Cancer, vol. 47, no. 13, pp. 1919-1927, 2011.

[8] K. Metcalfe, H. T. Lynch, P. Ghadirian et al., "Contralateral breast cancer in BRCA1 and BRCA2 mutation carriers," Journal of Clinical Oncology, vol. 22, no. 12, pp. 2328-2335, 2004.

[9] D. G. R. Evans, A. Moran, R. Hartley et al., "Long-term outcomes of breast cancer in women aged 30 years or younger, based on family history, pathology and BRCA1/BRCA2/TP53 
status," British Journal of Cancer, vol. 102, no. 7, pp. 1091-1098, 2010.

[10] K. Metcalfe, S. Gershman, H. T. Lynch et al., "Predictors of contralateral breast cancer in BRCA1 and BRCA2 mutation carriers," British Journal of Cancer, vol. 104, no. 9, pp. 1384-1392, 2011.

[11] M. K. Graeser, C. Engel, K. Rhiem et al., "Contralateral breast cancer risk in BRCA1 and BRCA2 mutation carriers," Journal of Clinical Oncology, vol. 27, no. 35, pp. 5887-5892, 2009.

[12] D. Ford, D. F. Easton, M. Stratton et al., "Genetic heterogeneity and penetrance analysis of the BRCA1 and BRCA2 genes in breast cancer families," The American Journal of Human Genetics, vol. 62, no. 3, pp. 676-689, 1998.

[13] D. G. Evans, A. Shenton, E. Woodward, F. Lalloo, A. Howell, and E. R. Maher, "Penetrance estimates for BRCA1 and BRCA2 based on genetic testing in a Clinical Cancer Genetics service setting: risks of breast/ovarian cancer quoted should reflect the cancer burden in the family," BMC cancer, vol. 8, article 155, 2008.

[14] F. Lalloo and D. G. Evans, "Familial breast cancer," Clinical Genetics, vol. 82, no. 2, pp. 105-114, 2012.

[15] D. Thompson and D. F. Easton, "Breast Cancer Linkage Consortium. Cancer incidence in BRCA1 mutation carriers," Journal of the National Cancer Institute, vol. 94, no. 18, pp. 1358-1365, 2002.

[16] N. Mavaddat, S. Peock, D. Frost et al., "Cancer risks for BRCA1 and $B R C A 2$ mutation carriers: results from prospective analysis of EMBRACE," Journal of the National Cancer Institute, vol. 105, no. 11, pp. 812-822, 2013.

[17] J. Gronwald, A. Robidoux, C. Kim-Sing et al., "Duration of tamoxifen use and the risk of contralateral breast cancer in BRCA1 and BRCA2 mutation carriers," Breast Cancer Research and Treatment, vol. 146, no. 2, pp. 421-427, 2014.

[18] A. Chompret, L. Brugières, M. Ronsin et al., "P53 germline mutations in childhood cancers and cancer risk for carrier individuals," British Journal of Cancer, vol. 82, no. 12, pp. 19321937, 2000.

[19] S. Masciari, D. A. Dillon, M. Rath et al., "Breast cancer phenotype in women with TP53 germline mutations: a LiFraumeni syndrome consortium effort," Breast Cancer Research and Treatment, vol. 133, no. 3, pp. 1125-1130, 2012.

[20] A. S. Reiner, E. M. John, J. D. Brooks et al., "Risk of asynchronous contralateral breast cancer in noncarriers of BRCA1 and $B R C A 2$ mutations with a family history of breast cancer: a report from the women's environmental cancer and radiation epidemiology study," Journal of Clinical Oncology, vol. 31, no. 4, pp. 433-439, 2013.

[21] S. K. McDonnell, D. J. Schaid, J. L. Myers et al., "Efficacy of contralateral prophylactic mastectomy in women with a personal and family history of breast cancer," Journal of Clinical Oncology, vol. 19, no. 19, pp. 3938-3943, 2001.

[22] A. K. Arrington, S. L. Jarosek, B. A. Virnig, E. B. Habermann, and T. M. Tuttle, "Patient and surgeon characteristics associated with increased use of contralateral prophylactic mastectomy in patients with breast cancer," Annals of Surgical Oncology, vol. 16, no. 10, pp. 2697-2704, 2009.

[23] J. L. Bernstein, R. H. Lapinski, S. S. Thakore, J. T. Doucette, and W. D. Thompson, "The descriptive epidemiology of second primary breast cancer," Epidemiology, vol. 14, no. 5, pp. 552-558, 2003.

[24] Z. Mitri, T. Constantine, and R. O’Regan, “The HER2 receptor in breast cancer: pathophysiology, clinical use, and new advances in therapy," Chemotherapy Research and Practice, vol. 2012, Article ID 743193, 7 pages, 2012.

[25] M. J. Piccart-Gebhart, M. Procter, B. Leyland-Jones et al., "Trastuzumab after adjuvant chemotherapy in HER2-positive breast cancer," New England Journal of Medicine, vol. 353, no. 16, pp. 1659-1672, 2005.

[26] L. Gianni, U. Dafni, R. D. Gelber et al., "Treatment with trastuzumab for 1 year after adjuvant chemotherapy in patients with HER2-positive early breast cancer: a 4-year follow-up of a randomised controlled trial," The Lancet Oncology, vol. 12, no. 3, pp. 236-244, 2011.

[27] B. S. Saltzman, K. E. Malone, J. A. McDougall, J. R. Daling, and C. I. Li, "Estrogen receptor, progesterone receptor, and HER2neu expression in first primary breast cancers and risk of second primary contralateral breast cancer," Breast Cancer Research and Treatment, vol. 135, no. 3, pp. 849-855, 2012.

[28] J. Cuzick, I. Sestak, J. F. Forbes et al., "Anastrozole for prevention of breast cancer in high-risk postmenopausal women (IBIS-II): an international, double-blind, randomised placebo-controlled trial," The Lancet, vol. 383, no. 9922, pp. 1041-1048, 2014.

[29] J. Cuzick, I. Sestak, M. Baum et al., "Effect of anastrozole and tamoxifen as adjuvant treatment for early-stage breast cancer: 10-year analysis of the ATAC trial," The Lancet Oncology, vol. 11, no. 12, pp. 1135-1141, 2010.

[30] Early Breast Cancer Trialists' Collaborative Group (EBCTCG), "Effects of chemotherapy and hormonal therapy for early breast cancer on recurrence and 15-year survival: an overview of the randomised trials," The Lancet, vol. 365, no. 9472, pp. 1687-1717, 2005.

[31] J. L. Bernstein, D. C. Thomas, R. E. Shore et al., "Contralateral breast cancer after radiotherapy among BRCA1 and BRCA2 mutation carriers: a WECARE Study Report," European Journal of Cancer, vol. 49, no. 14, pp. 2979-2985, 2013.

[32] R. Plummer, "Poly(ADP-ribose) polymerase inhibition: a new direction for BRCA and triple-negative breast cancer?" Breast Cancer Research, vol. 13, no. 4, article 218, 2011.

[33] A. Tutt, M. Robson, J. E. Garber et al., "Oral poly(ADPribose) polymerase inhibitor olaparib in patients with BRCA1 or BRCA2 mutations and advanced breast cancer: a proof-ofconcept trial," The Lancet, vol. 376, no. 9737, pp. 235-244, 2010.

[34] N. N. Basu, L. Barr, D. G. Evans, and G. L. Ross, “Threshold for genetic testing in women with breast cancer needs to be determined," British Medical Journal, vol. 348, Article ID g1863, 2014.

[35] D. G. Evans, J. Barwell, D. M. Eccles et al., “The Angelina Jolie effect: how high celebrity profile can have a major effect on provision of cancer related services," Breast Cancer Research, vol. 16, no. 5, article 442, 2014.

[36] S. M. Domchek, T. M. Friebel, C. F. Singer et al., "Association of risk-reducing surgery in BRCA1 or BRCA2 mutation carriers with cancer risk and mortality," JAMA-Journal of the American Medical Association, vol. 304, no. 9, pp. 967-975, 2010.

[37] L. Lostumbo, N. E. Carbine, and J. Wallace, "Prophylactic mastectomy for the prevention of breast cancer," Cochrane Database of Systematic Reviews, vol. 11, Article ID CD002748, 2010.

[38] K. Metcalfe, S. Gershman, P. Ghadirian et al., "Contralateral mastectomy and survival after breast cancer in carriers of BRCA1 and BRCA2 mutations: retrospective analysis," British Medical Journal, vol. 348, article g226, 2014. 
[39] D. G. R. Evans, S. L. Ingham, A. Baildam et al., "Contralateral mastectomy improves survival in women with BRCA1/2associated breast cancer," Breast Cancer Research and Treatment, vol. 140, no. 1, pp. 135-142, 2013.

[40] T. C. van Sprundel, M. K. Schmidt, M. A. Rookus et al., "Risk reduction of contralateral breast cancer and survival after contralateral prophylactic mastectomy in BRCA1 or BRCA2 mutation carriers," British Journal of Cancer, vol. 93, no. 3, pp. 287-292, 2005.

[41] S. B. Zeichner, A. L. Ruiz, N. J. Markward, and E. Rodriguez, "Improved long-term survival with contralateral prophylactic mastectomy among young women," Asian Pacific Journal of Cancer Prevention, vol. 15, no. 3, pp. 1155-1162, 2014.

[42] I. Bedrosian, C. Y. Hu, and G. J. Chang, "Population-based study of mastectomy and survival outcomes of breast cancer patients," Journal of the National Cancer Institute, vol. 102, no. 6, pp. 401409, 2010.

[43] P. R. Portschy, K. M. Kuntz, and T. M. Tuttle, "Survival outcomes after contralateral prophylactic mastectomy: a decision analysis," Journal of the National Cancer Institute, vol. 106, no. 8, Article ID dju160, 2014.

[44] T. A. King, R. Sakr, S. Patil et al., "Clinical management factors contribute to the decision for contralateral prophylactic mastectomy," Journal of Clinical Oncology, vol. 29, no. 16, pp. 2158-2164, 2011.

[45] A. Ashfaq, L. J. McGhan, B. A. Pockaj et al., "Impact of breast reconstruction on the decision to undergo contralateral prophylactic mastectomy," Annals of Surgical Oncology, vol. 21, no. 9, pp. 2934-2940, 2014.

[46] M. E. Miller, T. Czechura, B. Martz et al., "Operative risks associated with contralateral prophylactic mastectomy: a single institution experience," Annals of Surgical Oncology, vol. 20, no. 13, pp. 4113-4120, 2013.

[47] M. A. Crosby, P. B. Garvey, J. C. Selber et al., "Reconstructive outcomes in patients undergoing contralateral prophylactic mastectomy," Plastic and Reconstructive Surgery, vol. 128, no. 5, pp. 1025-1033, 2011.

[48] W.-B. Zhou, X.-A. Liu, J.-C. Dai, and S. Wang, "Meta-analysis of sentinel lymph node biopsy at the time of prophylactic mastectomy of the breast," Canadian Journal of Surgery, vol. 54, no. 5, pp. 300-306, 2011.

[49] R. E. Mansel, L. Fallowfield, M. Kissin et al., "Randomized multicenter trial of sentinel node biopsy versus standard axillary treatment in operable breast cancer: the ALMANAC trial," Journal of the National Cancer Institute, vol. 98, no. 9, pp. 599609, 2006.

[50] N. K. Kuwajerwala, N. S. Dekhne, P. A. Pentiak et al., "Sentinel lymph node biopsy in contralateral prophylactic mastectomy: are we overtreating? Experience at a tertiary care hospital," Clinical Breast Cancer, vol. 13, no. 4, pp. 287-291, 2013.

[51] V. R. Grann, P. R. Patel, J. S. Jacobson et al., "Comparative effectiveness of screening and prevention strategies among BRCA1/2-affected mutation carriers," Breast Cancer Research and Treatment, vol. 125, no. 3, pp. 837-847, 2011.

[52] D. Schrag, K. M. Kuntz, J. E. Garber, and J. C. Weeks, "Life expectancy gains from cancer prevention strategies for women with breast cancer and BRCA1 or BRCA2 mutations," Journal of the American Medical Association, vol. 283, no. 5, pp. 617-624, 2000.

[53] B. Zendejas, J. P. Moriarty, J. O’Byrne, A. C. Degnim, D. R. Farley, and J. C. Boughey, "Cost-effectiveness of contralateral prophylactic mastectomy versus routine surveillance in patients with unilateral breast cancer," Journal of Clinical Oncology, vol. 29, no. 22, pp. 2993-3000, 2011.

[54] A. A. Deshmukh, S. B. Cantor, M. A. Crosby et al., "Cost of contralateral prophylactic mastectomy," Annals of Surgical Oncology, vol. 21, no. 9, pp. 2823-2830, 2014.

[55] A. Roberts, M. Habibi, and K. D. Frick, "Cost-effectiveness of contralateral prophylactic mastectomy for prevention of contralateral breast cancer," Annals of Surgical Oncology, vol. 21, no. 7, pp. 2209-2217, 2014.

[56] S. T. Hawley, R. Jagsi, M. Morrow et al., "Social and clinical determinants of contralateral prophylactic mastectomy," JAMA Surgery, vol. 149, no. 6, pp. 582-589, 2014.

[57] A. Abbott, N. Rueth, S. Pappas-Varco, K. Kuntz, E. Kerr, and T. Tuttle, "Perceptions of contralateral breast cancer: an overestimation of risk," Annals of Surgical Oncology, vol. 18, no. 11, pp. 3129-3136, 2011.

[58] H. Beesley, C. Holcombe, S. L. Brown, and P. Salmon, "Risk, worry and cosmesis in decision-making for contralateral riskreducing mastectomy: analysis of 60 consecutive cases in a specialist breast unit," The Breast, vol. 22, no. 2, pp. 179-184, 2013.

[59] J. A. Murphy, T. D. Milner, and J. M. O’Donoghue, "Contralateral risk-reducing mastectomy in sporadic breast cancer," The Lancet Oncology, vol. 14, no. 7, pp. e262-e269, 2013. 


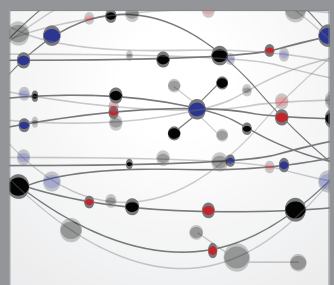

The Scientific World Journal
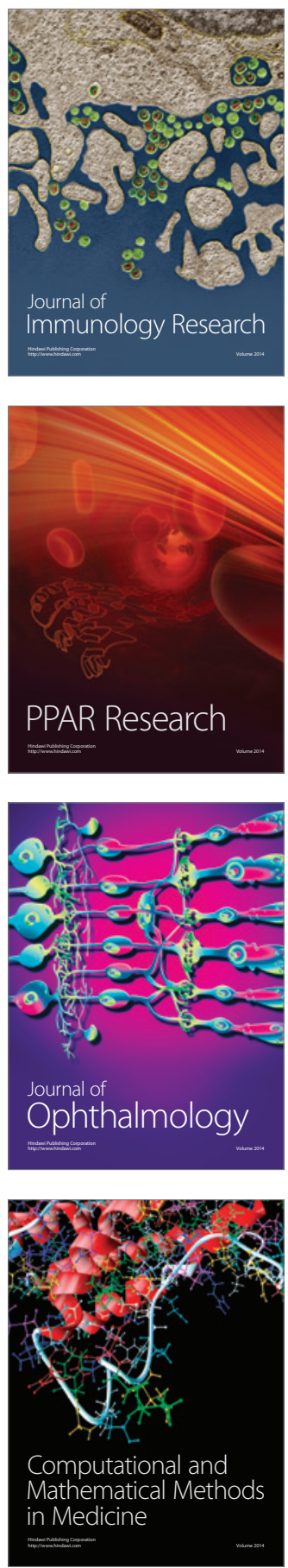

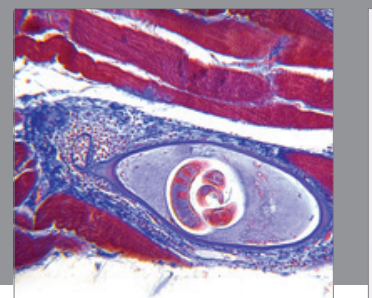

Gastroenterology

Research and Practice
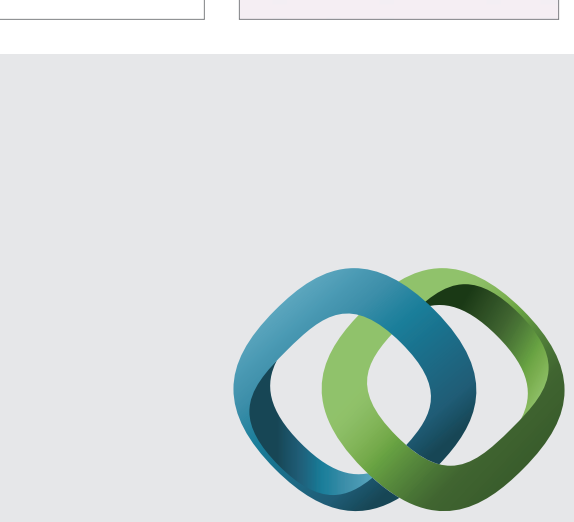

\section{Hindawi}

Submit your manuscripts at

http://www.hindawi.com
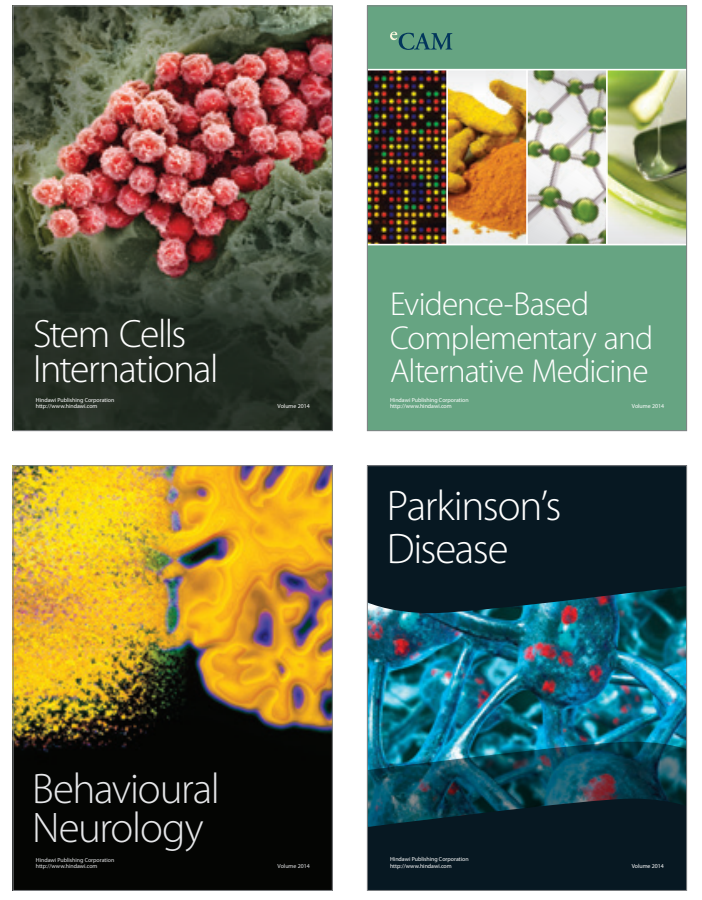
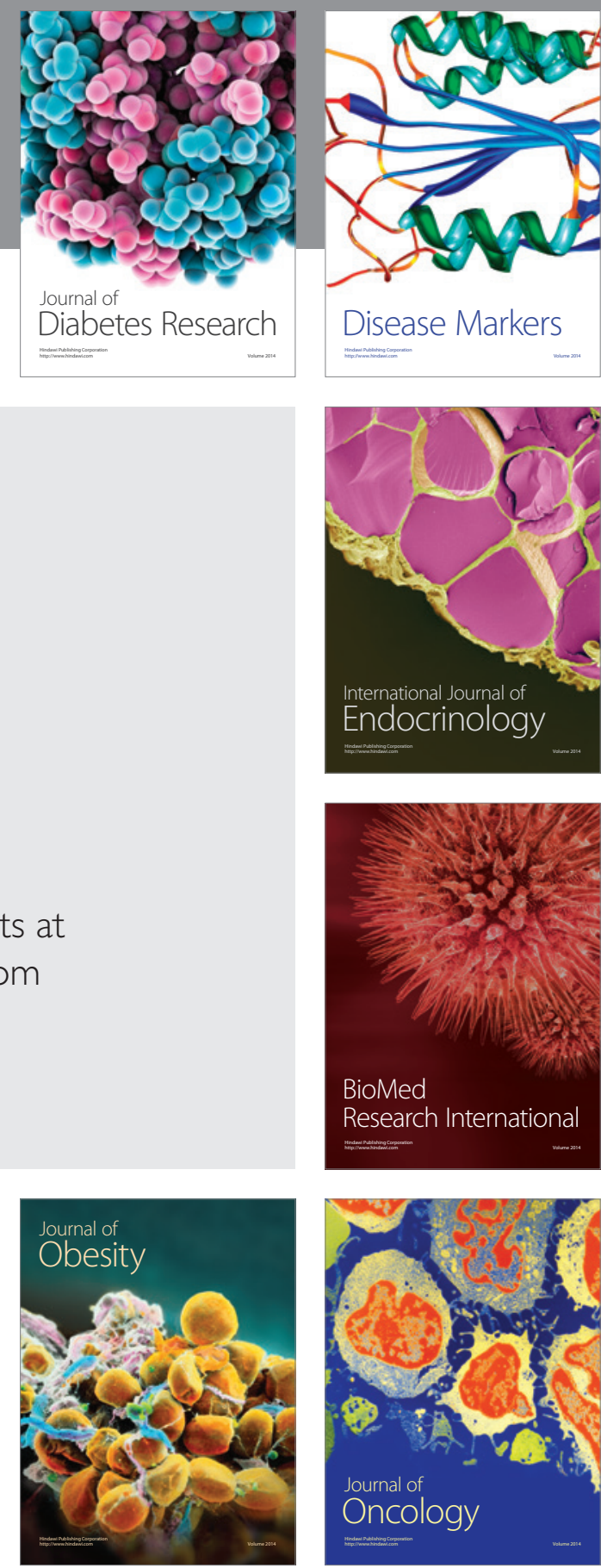

Disease Markers
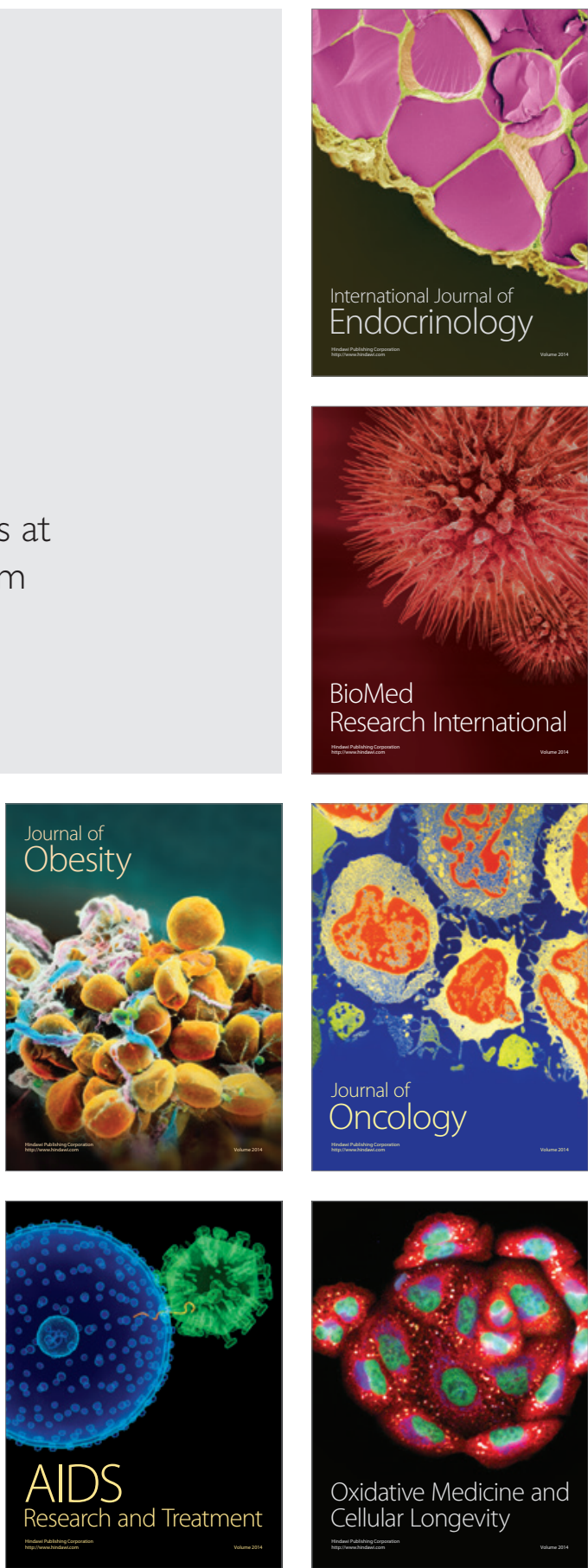